\title{
Efficiency Related Parameters in GPS based Vehicle Tracking System Working for an Organization
}

\author{
Rajeev Kumar, \\ Research Scholar \\ Bhagwant University, Ajmer Rajasthan India
}

\author{
Harsh Dev, PhD. \\ Professor and Director \\ Pranveer Singh Institute of Technology \\ Kanpur Uttar Pradesh India
}

\begin{abstract}
Tracking vehicles can be the result of the requirement to keep track of a fleet or even simply to keep track of personal vehicle. In modern time where vehicle thefts are common, a GPS vehicle tracking system can come in helpful almost everywhere. This is a fast growing service enabling field where contribution is done from the side of various national and international service providers. This paper entails the detail of parameters by which service providers can provide the efficient services to propagate the overall benefits of GPS vehicle tracking system. The performance of system is based on parameters related with system or environment. Some parameters are known and involved in the distribution of services of implemented systems. The working of GPS based vehicle tracking systems is continuously improving by considering the known parameters. The best performance of the systems is still awaited and some inefficient situations occurred during working with the systems. The considerable additional factors which should be analyzed and kept in mind when GPS based vehicle tracking systems are being deployed. The efficiency of GPS based vehicle tracking systems can be improved by considering proposed parameters.
\end{abstract}

\section{Keywords}

Global Positioning System, Trilateration, LBS etc.

\section{INTRODUCTION}

Tracking of vehicle has been emerged as a leading medium of information fetching from the related entities that are available at different geographical locations. It takes just a click and the communication reaches to the recipient's system that is also connected with the GPS network. While addressing the efficiency issues in the GPS based vehicle tracking systems due to environmental factors, we have observed that in most of organization's owners believes that they are providing best services by considering the existing parameters [1-3] because they have deployed the system with proper mechanism for overcoming the inefficient conditions and following efficient conditions. Parameters whose work is actually to provide the favorable results always in consideration but the silent parameters which affect the performance of tracking are main threat to be identified for technical persons. The tracking authorities are really interested to implement the best solutions and this paper is providing essential guidelines for getting best output in order to track the desired entity. In the Fig. 1 a Layout of GPS based vehicle tracking system is shown. Our effort is to recognize the additional parameters so that the service providers can improve the performance during tracking.
Tracking The Global Positioning System (GPS) is an assemblage of $27^{*}$ Earth-orbiting satellites [1]. The U.S. military developed and implemented this satellite network as a military navigation system but, soon opened it up to everyone. Each of these 3500-4500-pound solar-powered satellites circles the globe at about of 12500 miles, making two complete rotation every day. The orbits are arranged so that at anytime, anywhere on the earth there are at least four satellites appearing in the sky. The work of GPS receiver is, to locate four or more of these satellites, outline the distance to each, and utilize this information to figure out its own location. This operation is based on Trilateration a simple mathematical principle. For location calculation GPS receiver has to know the location of at least three satellites above you and the distance between you and each of those three satellites.

Broadly GPS based vehicle tracking systems can be partitioned into two main parts -In-vehicle unit and tracking station or monitoring server. In vehicle unit is a major part of tracking systems and installed into vehicles for fetching location and other required information. This is also responsible to send the collected information to the tracking sever located anywhere [2]. The needed information processing is done by different components of in-vehicle unit. Data Transceiver is used to transfer the information to Monitoring server. For real time tracking of vehicles reliable data transmission is needed. GSM network is utilized for transferring extracted and processed information to remote server. Fetched data (Location data) is transferred through Microcontroller. The processed information through Microcontroller transmitted to remote location through GSM modem. The Microcontroller is working as CPU for whole process. Instructions are required to operate Microcontroller; these instructions are provided to Microcontroller by writing the software. Other component is tracking server partitioned into four parts, Required H/w design, Data base for authorize access, Communication S/W, Web interface for user.

The paper is classified into nine sections. Section 1 is introduction, and basics of GPS to use in tracking assignment. In this section the existing parameter which is related with the performance of GPS based vehicle tracking systems are explained. Section 2 is describing about the classification of parameters. Section 3 is describing about the impact of various factors on working of GPS based vehicle tracking systems. Section 4 is describing about to enlarge the existing set of parameters for better use in working scenario. Section 5 is telling about authors contribution. Section 6 is dedicated to conclude the attempt made by authors in this paper. Section 7 is explaining about the future work. Section 8 is about acknowledgement and Section 9 is about the list of references and books. 


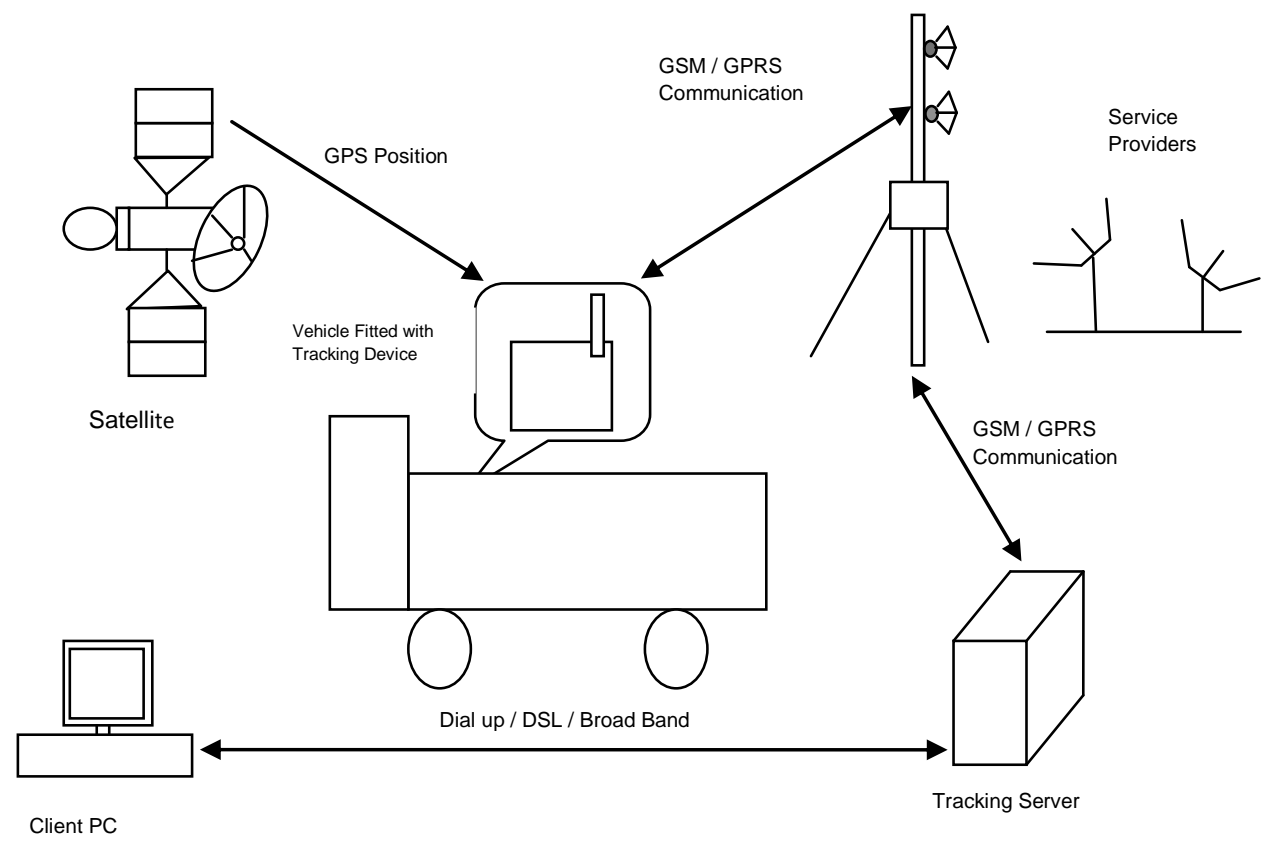

Fig 1: Working of GPS based Vehicle Tracking Systems

\section{CLASSIFICATION OF PARAMETERS}

The Frame work of LBS is utilized by GPS based vehicle tracking system. The context background of LBS is a powerful location identification technique in case of tracking the vehicle by GPS based systems [3]. On the bases of this particular background some critical parameters can be identified which are directly related with the efficiency of GPS based vehicle tracking system shown in figure 2 .

According this existing approach environmental context for extracting location information is essential. The Users use devices for extracting information and interaction between user and device dependent on nature of environment. The exact location information is affected by that background (Rural or Urban). Mobile Cell-id provided by Location information used for physical identification of tracking object.
Technological context is another critical parameter because by using the best suitable technology location information can be fetched in optimized manner. A major role played by technology tailored in extracting device during location information identification of tracking object. Due to the joint nature of complete GPS based vehicle systems existing network can also be considered into technological context. Optimized networks are required as transfer medium during information extraction and processing. In working scenario of GPS based vehicle tracking systems user can also be considered another parameter. Users devote in the system through personal characteristics, preferences, interaction and ability of communicating device. After analyzing existing working environment of GPS based vehicle tracking system the countable parameters are reflected by figure 2[3]. 


\section{Environment}

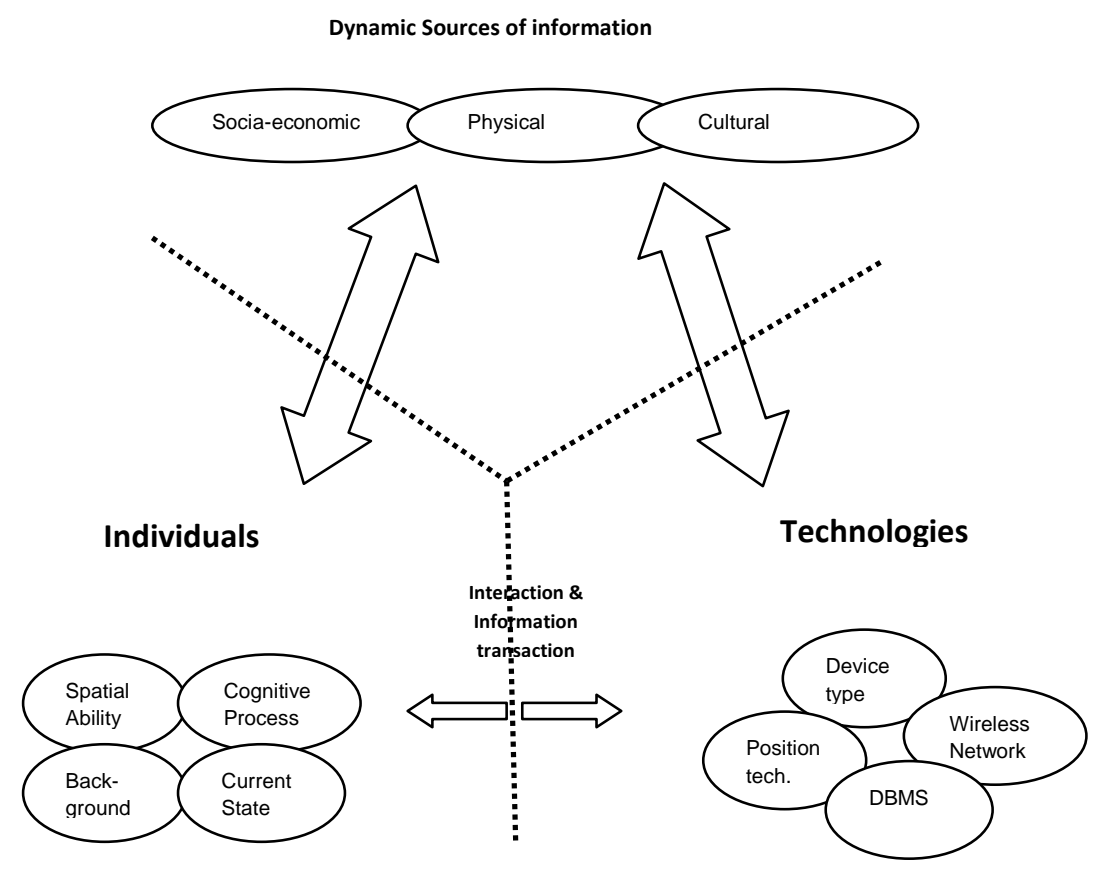

Fig 2: Existing Parameters in GPS Support (Source Allan Brimicombe)

\section{ANALYSIS OF EXISTING PARAMETERS}

In this section we are explaining the involvement of critical parameters for giving the performance in GPS based vehicle tracking systems which is analyzed and verified through existing work during recent past years. The work [4] has added the knowledge about the generation of location based services. After this paper the used technologies like positioning techniques and user interaction grasped and smoothen by the service providers so that the main objective of clients can be achieved. The work [5] proposed a cloud based approach for improving the performance and enabling the smoothness in the working of GPS based vehicle tracking systems.

\subsection{Location Based Services}

LBS is having greater impact in the implementation of GPS based vehicle tracking system by providing mobile by mobile information [6] through mobile positioning in a desired scenario.

LBS work as a shared point between GIS, internet, communication network and national information and communication technologies. The components of LBS are communication links, service providers, data and content provider and device of positioning. The execution of LBS is fully dependent on above components. LBS are having a small history behind the existing architecture. The era (19972001) is called the first generation and after that second generation is going on [4]. The first generation was based on link services with MPC/GLMC architecture. In second Generation the concept of navigation was used [7]. A crucial role is played by positioning techniques in navigation system.

\subsection{Environmental Context}

GPS based vehicle tracking systems dealt with environmental variables like Sensing of signals emitted by object based on moments which are optical in nature, detecting reflection of signals emitted from a base station called pinging, detection of specific transponder signal called tagging, access to GPS signal (satellites) and mechanisms for data storing and/or data transmission to central processing unit [8]. These above specified variables are managed and controlled by various components dedicated to GPS based vehicle tracking systems.

\subsection{Technological Context}

GPS based vehicle tracking systems hold the technology according implementation like High GPS (Based on more than four satellites [9]), Low GPS (Based on less than three satellites), and No GPS (No satellite). The technological implementation also dedicated to Effective Crew/Fleet Planning, Patronage of Public Transport System, Tracking \& Monitoring, and Increase in productivity, Reduction in travel time, Reduction in accidents.

\subsection{User Context}

The best utilization of GPS based vehicle tracking system dependent on nature of the user. Performance is boiled by technical expertise of the user. GPS based vehicle tracking system is handled efficiently by fully professional user. All the features of system analyzed and compiled by this kind of users [9]. Some popular features are used by the partially professional users. This kind of users can be interested in report generation only. The third category of users is having no knowledge of GPS based vehicle tracking systems and system is hired for security purposes.

\section{Our Work}

The views of technical persons were collected from different organizations (Nearly 10) who are dealing with the deployment of GPS based vehicle tracking systems through survey in the duration of 6 months (17/08/2012-17/02/2013) related with the parameters which can be critical with respect in performance of deployed systems. Some existing parameters verified by the technical persons and owners but some new parameters also outlined. The deployment handlers 
were in agreement of that some parameters are really related with the performance of GPS based systems but some parameters can be ignored partially or fully (see table 1). Such organizations are handling 50 to 1500 clients under one deployed GPS based vehicle tracking systems. These clients are dealing in the business of Carriers, Trucks, Trails, Tankers, Taxies, and Oil etc. Most of the questions in survey are based on existing parameters and the parameter identified during our research

Table 1. Result of Survey Conducted with Organizations

\begin{tabular}{|l|c|l|c|}
\hline Parameters & $\begin{array}{l}\text { Fully Related with } \\
\text { Efficiency }\end{array}$ & $\begin{array}{l}\text { Partially related with } \\
\text { Efficiency }\end{array}$ & Can be overlooked \\
\hline Physical location & $\mathbf{x}$ & $\checkmark$ & $\mathbf{x}$ \\
\hline Socio economic structure & $\mathbf{x}$ & $\mathbf{x}$ & $\checkmark$ \\
\hline Cultural environment & $\checkmark$ & $\mathbf{x}$ & $\mathbf{x}$ \\
\hline Spatial ability & $\checkmark$ & $\mathbf{x}$ & $\mathbf{x}$ \\
\hline Cognitive process & $\checkmark$ & $\mathbf{x}$ & $\mathbf{x}$ \\
\hline Environmental background & $\checkmark$ & $\mathbf{x}$ & $\mathbf{x}$ \\
\hline Current state & $\checkmark$ & $\mathbf{x}$ & $\mathbf{x}$ \\
\hline Device type & $\checkmark$ & $\mathbf{x}$ & $\mathbf{x}$ \\
\hline Wireless network & $\checkmark$ & $\mathbf{x}$ & $\mathbf{x}$ \\
\hline Position technology & $\checkmark$ & $\mathbf{x}$ \\
\hline DBMS & $\checkmark$ & $\mathbf{x}$ & $\mathbf{x}$ \\
\hline Electric wires & $\checkmark$ & $\mathbf{x}$ & $\mathbf{x}$ \\
\hline Floors of building & $\checkmark$ & $\mathbf{x}$ & $\mathbf{x}$ \\
\hline $\begin{array}{l}\text { No of satellites(For covering } \\
\text { maximum speed) }\end{array}$ & $\checkmark$ & $\mathbf{x}$ \\
\hline Knowledge of user & $\checkmark$ & \\
\hline
\end{tabular}

\section{OUR PROPOSED PARAMETERS}

In this paper we are proposing some additional parameters which are crucial with respect in efficiency of GPS based vehicle tracking systems.

1. If the organization is going to deploy any GPS based system than user should be trained so that all the features of deployed system are identified in such a manner that they can utilize the system to generate productive scenario.

2. The deployment of GPS based vehicle tracking system must be according the structure of working building. GPS does not work in basement. The floor of implementation must be according guidelines.

3. The proximity of electric wires must be analyzed during deployment of the GPS based vehicle tracking systems so that the best performance got.

4. In vehicle tracking, coverage of speed for vehicle is also a measurable factor in fetching the information through GPS based vehicle tracking systems, therefore maximum satellite support should inherent for fetching the efficient information.

\section{CONCLUSION}

In this research related effort we have analyzed the existing support parameters for GPS based vehicle tracking systems. In our survey the questions were asked related with existence of new parameters and the views of technical persons about existing parameters crosschecked. The limitation and advantages of these parameters also discussed with technical persons. The authors brought out required changes in the set of existing parameters for improving the working of GPS based vehicle tracking systems. This paper is survey based research attempt extending the range of efficiency related parameters for service providers. The best performance can be achieved with the help of crucial parameters proposed by the authors for improving the efficiency of GPS based vehicle tracking systems. These proposed parameters are based on extraction of history, study and survey.

\section{FUTURE DIRECTIONS}

In future this research attempt will be verified through the clients of the organizations which are using the GPS based vehicle tracking systems. The merging of verified parameters into existing parameters will be done for evaluation of efficiency, achieved after considering proposed parameters by using a working software tool. The framework will be designed after verification for the technical implementation of these proposed parameters for significant improvement in the performance of the GPS based vehicle tracking systems. The performance of system will be optimized by using the dynamic programming approach.

\section{ACKNOWLEDGEMENT}

We extend our sincere thanks to various technical members who shared valuable knowledge of GPS based vehicle tracking systems and dealing various efficiency related issues of users. This discussion helped us to carry on our work of this paper.

\section{REFERENCES}

[1]. Akinode J.L1, Alawode A.J2 And Ojuawo O.O3. Nigeria, "Improving National Security using GPS Trackinsystem Technology. Proceedings of the 1st International Technology, Educationand Environment Conference(c) African Society for Scientific Research (ASSR).

[2]. Ambade Shruti Dinkar , S.A.Sackh, "Design and implementation of Vehicle tracking system using GPS", Journal of Information Engineering and Applications, Vol 1,No.3,2011 
[3]. Allan Brimicombe, Chao Li, "Location Based Services and Geo-information Engineering", Willey BlackWell A John Willey \& Sons Ltd. Publications, First Impression 2009.

[4]. Moein Mohammadi, Ehasan Molaei, Ali Naserasadi, "A Survey on Location Based Services and Positioning Techniques", International Journal of Computer Applications, volume 24-No 5, June 2011.

[5]. Albert Alexe, R.Ezhilarasie, "Cloud Computing Based Vehicle Tracking Information systems", IJCST Vol 2, Issue 1, March 2011.

[6]. Barkuus, Louise, "Location Based Services for Mobile Telephony". Intel Corporation, 2003,
[7]. Wang, Shu, Jungwon Min and Byung K. Yi, "Location based services for mobiles: Technology and Standards". IEEE international conference on ommunication (ICC) Beijing, China; 2008,

[8]. Siegrist, D., Clivaz, C., Hunziker, M. \& Iten, S. (eds.) (2006). "Exploring the Nature of Management", Proceedings of the Third International Conference on Monitoring and Management of Visitor Flows in Recreational and Protected Areas. University of Applied Sciences Rapperswil, Switzerland, 13-17 September 2006. Rapperswil

[9]. ITG Telemetics Pvt. Ltd., The best pearl hight-1, Netaji Subash Palace, New Delhi 